TRANSACTIONS OF THE

AMERICAN MATHEMATICAL SOCIETY

Volume 188, Issue 2, 1974

\title{
ULTRAFILTER MAPPINGS AND THEIR DEDEKIND CUTS
}

\author{
BY
}

\section{ANDREAS BLASS}

ABSTRACT. Let $D$ be an ultrafilter on the set $N$ of natural numbers. To each function $p: N \rightarrow N$ and each ultrafilter $E$ that is mapped to $D$ by $p$, we associate a Dedekind cut in the ultrapower $D$-prod $N$. We characterize, in terms of rather simple closure conditions, the cuts obtainable in this manner when various restrictions are imposed on $E$ and $p$. These results imply existence theorems, some known and some new, for various special kinds of ultrafilters and maps.

Although some of what we say can be generalized to larger cardinals, we shall confine our attention to ultrafilters on a countable set, which we may take to be the set $N$ of natural numbers. It will be convenient to identify $N \times N$ with $N$ by means of one of the standard pairing functions; thus, the projections, $\pi_{1}$ and $\pi_{2}$, from $N \times N$ to $N$ may be viewed as maps from $N$ to $N$.

If $E$ is an ultrafilter on $N$, we define an equivalence relation on the functions from $N$ to $N$ by declaring two functions to be equal mod $E$ iff their restrictions to some set in $E$ are the same. The equivalence class $[f]$ of a function $f$ is called its germ (more precisely, its $E_{-g e r m}[f]_{E}$ ), and the set of all germs is the ultrapower $E$-prod $N$. All relations and operations defined on $N$ have natural extensions making $E$-prod $N$ an elementary extension of $N$, provided we identify the germs of constant functions with the values of these functions (see [8]).

A function $p: N \rightarrow N$ maps $E$ to the ultrafilter $p(E)=\left\{A \subseteq N \mid p^{-1}(A) \in E\right\}$; $p(E)$ depends only on $E$ and the $E$-germ of $p$. If $p$ maps $E$ to $D$, we write $p: E \rightarrow D$. If, in addition, $p$ is one-to-one on some set in $E$, then we call $p$ an isomorpbism from $E$ to $D$ and write $p: E \cong D$. In this case, there is a permutation of $N$ equal $\bmod E$ to $f$, and there is an isomorphism $g: D \cong E$, unique $\bmod D$, such that $f \circ g$ and $g \circ f$ are equal, $\bmod D$ and $E$ respectively, to the identity. It is easy to check that the composite of two isomorphisms is again an isomorphism.

If we replace a map $p: E \rightarrow D$ by another with the same germ, or if we compose $p$ with an isomorphism $E^{\prime} \cong E$, all the properties of $p$ in which we shall be

Received by the editors July 31, 1972. $04 \Lambda 30$.

AMS (MOS) subject classifications (1970). Primary 04A20; Secondary 02H13, 02H20,

Key words and phrases. Ultrafilters, $P$-points, Ramsey ultrafilters, ultrapowers, Dedekind cuts. 
interested remain unchanged. By means of such modifications, we can convert any $p$ into $\pi_{1}$. Whenever we call a map $p$, the reader should visualize it as $\pi_{1}: N \times N$ $\rightarrow N$. We call the sets $p^{-1}\{n\}$ fibers, and we say that a function is fiberwise constant on a set $A$ iff its restriction to $A \cap p^{-1}\{n\}$ is constant for each $n$ (the constant may depend on $n$ ). The notion of fiberwise one-to-one is defined analogously.

We call a map $p: E \rightarrow D$ irreducible $($ on $E$ ) iff, whenever $p=f \circ g \bmod E$, either $g: E \cong g(E)$ or $f: g(E) \cong D$.

Lemma. A necessary and sufficient condition for $p$ to be irreducible on $E$ is that every function $b$ be fiberwise one-to-one or fiberwise constant on some set in $E$.

Proof. Necessity: For any given $b$, define $g(x)=(b(x), p(x)), f(x)=\pi_{2}(x)$, so that $p=f \circ g$. If $g: E \cong g(E)$, then $g$ is one-to-one on a set in $E$, which clearly implies that $b$ is fiberwise one-to-one on the same set. On the other hand, if $f: g(E) \cong D$, then $f$ is one-to-one on a set $B \in g(E)$. By definition of $f$ and $g$, it follows that $b(x)=b(y)$ whenever $g(x) \in B, g(y) \in B$, and $p(x)=p(y)$. This means that $b$ is fiberwise constant on $g^{-1}(B) \in E$.

Sufficiency: Let $p=f \circ g \bmod E$, say $p=f \circ g$ on $A \in E$. Then the restriction $g \mid A$ never takes the same value in different fibers. If $g$ is fiberwise oneto-one on a set $B \in E$, then $g$ is one-to-one on $A \cap B \in E$, so $g: E \cong g(E)$. On the other hand, if $g$ is fiberwise constant on some $B \in E$, then there is a map $k$ such that $g=k \circ p$ on $B$. Thus $g=k \circ f \circ g$ on $A \cap B$ and $f$ is one-to-one on $g(A \cap B) \in g(E)$, so $f: g(E) \cong D$.

We call a nonprincipal ultrafilter $E$ a Ramsey ultrafilter iff it satisfies one, hence all, of the following three equivalent conditions:

(1) A constant function is irreducible on $E$.

(2) Every function on $N$ is one-to-one or constant on some set in $E$.

(3) For every partition of $[N]^{2}$, the set of unordered pairs from $N$, into two parts, there is an $A \in E$ such that $[A]^{2}$ is included in one of the parts.

(The only nontrivial implication, $(2) \Rightarrow(3)$, is due to Kunen; a proof is given in [3].)

By weakening conditions (2) and (3), we obtain two wider classes of ultrafilters. A nonprincipal ultrafilter $E$ is a $P$-point iff every function on $N$ is finite-to-one or constant on some set in $E$. An equivalent condition is that, for every countable subfamily $\left\{A_{n} \mid n \in N\right\}$ of $E$, there is a set $B \in E$ which is almost included in each $A_{n}$ in the sense that $B-A_{n}$ is finite for each $n$. It follows that any nonprincipal ultrafilter generated by an almost decreasing sequence $\left\{A_{a} \mid a<\boldsymbol{X}_{1}\right\}$ of length $\boldsymbol{K}_{1}\left(\boldsymbol{N}_{1}=\right.$ the first uncountable ordinal $)$ is a $P$-point. A nonprincipal ultrafilter $E$ is weakly Ramsey iff, for every partition of $[N]^{2}$ into 
three parts, there is an $A \in E$ such that $[A]^{2}$ is included in the union of two of the parts. $P$-points have been rather extensively studied (see [2], [3], [4], [5], [10], [11]), but the notion of weakly Ramsey ultrafilter appears to be new. Of course every Ramsey ultrafilter is a $P$-point; the converse is false if the continuum hypothesis (henceforth abbreviated $\mathrm{CH}$ ) holds (see [3]). It is clear also that all Ramsey ultrafilters are weakly Ramsey, and we shall show later that all weakly Ramsey ultrafilters are $P$-points. If we assume $\mathrm{CH}$, then Theorems 2 and 4 below imply that neither converse holds.

A partition $(S, L)$ of a linearly ordered set is a (Dedekind) cut iff no element of $L$ precedes any element of $S$. ( $S$ and $L$ stand for "small". and "large".) A cut $(S, L)$ of $D$-prod $N$ is proper iff $L$ is nonempty and $S$ contäins the germs of all constant maps (the standard part of $D$-prod $N$ ).

For any $A \subseteq N$ and any $p: N \rightarrow N$, we define the cardinality function of $A$ (relative to $p$ ) by

$$
c_{A}(n)=\text { cardinality of } A \cap p^{-1}\{n\} .
$$

If $p: E \rightarrow D$, then the $D$-germs of the cardinality functions of the sets in $E$, and all larger $D$-germs, constitute the upper part $L$ of a cut $(S, L)$ of $D$-prod $N$, which we call the cut associated to $p$ and $E$. (Notice that, according to our definitions, if $c_{A}(n)$ is infinite for some $n$ then $c_{A}$ has no germ, so it makes no contribution to $L$; it is entirely possible for $L$ to be empty.) The cut associated to $p$ and $E$ is proper iff $p$ is finite-to-one on some set in $E$ but not one-to-one on any set in $E$. To emphasize the similarity between our theorems, we shall state them only for proper cuts, postponing to the end of the paper the simpler case of improper cuts.

In each of the following theorems, $D$ is an ultrafilter on $N$, and $(S, L)$ is a proper cut in $D$-prod $N$. (Notice that the existence of a proper cut implies that $D$ is nonprincipal.)

Theorem 1. $(S, L)$ is the cut associated to some map of some ultrafilter to $D$ iff $S$ is closed under (the natural extension to $D$-prod $N$ of) addition.

Theorem 2. Assume CH. $(S, L)$ is the cut associated to some map of some $P$-point to $D$ iff $D$ is a $P$-point, $S$ is closed under addition, and every countable subset of $L$ bas a lower bound in $L$.

Theorem 3. Assume CH. $(S, L)$ is the cut associated to some irreducible map of some ultrafilter to $D$ iff $S$ is closed under multiplication and every countable subset of $L$ bas a lower bound in $L$.

Theorem 4. Assume CH. $(S, L)$ is the cut associated to some map of some weakly Ramsey ultrafilter to $D$ iff $D$ is Ramsey, $S$ is closed under exponentia. tion, and every countable subset of $L$ bas a lower bound in $L$. 
Before proving these theorems, we make a few remarks about them and deduce some consequences from them.

For proper cuts $(S, L)$, closure of $S$ under multiplication implies closure under addition, for $2 \in S$, so $a, b \in S \Rightarrow a+b \leq 2 \max \{a, b\} \in S$. Similarly, closure under exponentiation implies closure under multiplication.

In Theorems 2, 3, and 4, the continuum hypothesis is used only in the "if" part of the proof. It is reasonable to assume $\mathrm{CH}$ here because the very existence of $P$-points, weakly Ramsey ultrafilters, and irreducible maps has been proved only with the aid of $\mathrm{CH}$ or other special hypotheses such as Martin's axiom. See [1], [3], [11].

The condition, appearing in Theorems 2,3 , and 4, that every countable subset of $L$ have a lower bound in $L$, will be automatically satisfied if $S$ has a countable cofinal subset, because $D$-prod $N$ is $\kappa_{1}$-saturated (see [9]). For example, we may take $S$ to be the standard part of $D$-prod $N$. Using this choice of $S$, we find from Theorem 4 that if $\mathrm{CH}$ holds, there are weakly Ramsey ultrafilters that are not Ramsey.

The same choice of $S$ in Theorem 2 yields the known result [1], [10] that, if $\mathrm{CH}$ holds, then for every $P$-point $D$ there is a $P$-point $E$ and a map $p: E \rightarrow D$ that is not an isomorphism. Theorem 3 shows that every ultrafilter is the image of an irreducible map, provided $\mathrm{CH}$ holds. (This result was obtained by another method in [1]. The present approach yields finite-to-one irreducible maps, which the method of [1] did not.)

The conditions in Theorem 2 are substantially weaker than those in Theorem 4. In the first place, there are $P$-points $D$ that are not Ramsey, and, in the second place, there are cuts $(S, L)$ that satisfy the conditions of Theorem 2 but with $S$ not even closed under multiplication. (For example, let $a \in D-p r o d N$ be infinite, and let $S=\{x \in D$-prod $N \mid x<n a$ for some $n \in N\}$.) Hence, not every $P$-point is weakly Ramsey.

Many of the ultrafilter-theoretic concepts involved in our theorems have natural model-theoretic interpretations in terms of ultrapowers. Let us consider, for the moment, only those models that are elementary extensions of the standard model whose universe is $N$ and whose relations and functions are all the relations and functions on $N$. By abuse of language, we use the same symbol for a model and its universe. Since the model $N$ has built-in Skolem functions, the notions of submodel and elementary submodel coincide for the models under consideration. An element $b$ of model $B$ is said to generate $B$ over a submodel $A$ iff no proper submodel of $B$ includes $A \cup\{b\}$. For example, $D-\operatorname{prod} N$ is generated over its standard part by $[i]_{D}$ where $i$ is the identity function, and any model generated over $N$ by a single element is isomorphic to an ultrapower of $N$. Any map $p: E \rightarrow D$ induces an elementary embedding 


$$
p^{*}: D-p r o d N \rightarrow E-p r o d N:[f]_{D} \mapsto[f \circ p]_{E} ;
$$

$p^{*}$ depends only on $[p]_{E}$. The map $p$ is an isomorphism of ultrafilters iff $p^{*}$ is surjective and thus an isomorphism of models. The image of $p^{*}$ is cofinal in $E$-prod $N$ iff $p$ is finite-to-one on some set of $E$. Thus, $E$ is a $P$-point (resp. Ramsey ultrafilter) iff every nonstandard submodel of $E$-prod $N$ is cofinal in (resp. equal to) $E$-prod $N$. The map $p$ is irreducible iff no proper submodel of $E$-prod $N$ properly includes the image of $p^{*}$, i.e. iff $E-\operatorname{prod} N$ is a minimal extension of that image. An element $a \in D$-prod $N$ is in the upper half of the cut associated to $p$ and $E$ iff $p^{*}(a)$ is greater than some generator of $E \cdot \operatorname{prod} N$ over $p^{*}(D-\operatorname{prod} N)$.

This description of the cut associated to a map of ultrafilters suggests a definition of a cut associated to any elementary embedding $g: A \rightarrow B$ even if $A$ and $B$ are not ultrapowers of $N$. One can even consider models which are not elementary extensions of $N$, for example, arbitrary models of Peano arithmethic. It is not difficult to generalize Theorem 1 to this situation. Although it seems likely that analogs of some of our other theorems also hold in more general contexts, we do not pursue the matter further and turn instead to the proofs of our theorems.

Proof of Theorem 1. Suppose $(S, L)$ is the cut associated to $p: E \rightarrow D$, and suppose $[f]_{D}+[g]_{D} \in L$. By definition of associated cuts, there exists $A \in E$, $B \in D$ such that $c_{A}(n) \leq f(n)+g(n)$ for all $n \in B$. Thus, we can partition each of the fibers $A \cap p^{-1}\{n\}$ of $A$ into two pieces, $C_{n}$ and $C_{n}^{\prime}$, whose cardinalities are at most $f(n)$ and $g(n)$ respectively for $n \in B$. The sets $C=\bigcup_{n} C_{n}$ and $C^{\prime}=\bigcup_{n} C_{n}^{\prime}$ partition $A$, so one of them is in $E$. Since their cardinality functions are majorized on $B$ by $f$ and $g$ respectively, it follows that $[f]_{D}$ or $[g]_{D}$ is in $L$.

Conversely, let $(S, L)$ be any cut such that $S$ is closed under addition. We set $p=\pi_{1}: N \times N \rightarrow N$, and look for an ultrafilter $E$ on $N \times N$ such that $p: E \rightarrow D$ and $(S, L)$ is the associated cut. Let

$$
\begin{aligned}
& F_{1}=\left\{p^{-1}(A) \mid A \in D\right\}, \\
& F_{2}=\left\{\left.\{(x, y) \in N \times N \mid y<g(x)\}\right|_{g}: N \rightarrow N \text { and }[g]_{D} \in L\right\}, \\
& F_{3}=\left\{(N \times N)-X \mid\left[c_{X}\right]_{D} \in S\right\} .
\end{aligned}
$$

Note that each $F_{i}$ is closed under finite intersection (for $F_{3}$ we use the fact that $S$ is closed under addition), and that the intersection of three sets, one from each $F_{i}$, cannot be empty (because, if $[g] \in L,\left[c_{X}\right] \in S$, and $A \in D$, then $c_{X}$ cannot majorize $g$ on all of $A$ ). Thus, $F_{1} \cup F_{2} \cup F_{3}$ has the finite intersection property and can be extended to an ultrafilter $E . p(E)=D$ because $F_{1} \subseteq E$, and the associated cut is $(S, L)$ because $F_{2} \cup F_{3} \subseteq E$.

Proof of Theorem 2. Suppose $(S, L)$ is the cut associated to $p: E \rightarrow D$ and $E$ is a $P$-point. By Theorem $1, S$ is closed under addition. If $f: N \rightarrow N$, then $f \circ p$ is finite-to-one or constant on a set $A \in E$, so $f$ is finite-to-one or constant 
on $p(A) \in D$; as $D$ is nonprincipal and $f$ is arbitrary, we conclude that $D$ is a $P$-point. To prove the necessity of the remaining condition, let $\left\{\left[g_{i}\right]_{D} \mid i \in N\right\}$ be a countable subset of $L$. Thus, there are sets $A_{i} \in E$ and $B_{i} \in D$ such that $c_{A_{i}}(n) \leq g_{i}(n)$ for all $n \in B_{i}$. As $E$ is a $P$-point, there is a set $A \in E$ almost included in each $A_{i}$. Then $c_{A}(n) \leq c_{A_{i}}(n) \leq g_{i}(n)$ for all but finitely many $n \in B_{i}$, hence for all $n$ in a certain set in $D$ (as $D$ is nonprincipal). Therefore, $\left[c_{A}\right]$ is a lower bound in $L$ for the $\left[g_{i}\right]$ 's.

Conversely, let a $P$-point $D$ and a proper cut $(S, L)$ satisfying the condition of the theorem be given. We let $p=\pi_{1}: N \times N \rightarrow N$, and we construct a $P$-point $E$ on $N \times N$ such that $p(E)=D$ and $(S, L)$ is the associated cut. $E$ will be generated by an almost decreasing sequence $\left\{A_{a} \mid \alpha<\aleph_{1}\right\}$ (i.e. $\alpha<\beta \Rightarrow A_{\beta}-A_{a}$ is finite). As remarked above, this suffices to guarantee that $E$ is a $P$-point. Each $A_{a}$ will be large in the sense that $\left[c_{A_{a}}\right]_{D} \in L$; this suffices to gyarantee that $p(E)=D$ and $L$ includes the upper part of the associated cut. In addition, we impose the following requirements on the $A_{a}$ 's. First, for each $X \subseteq N \times N$ :

$$
\text { Either } X \text { or }(N \times N)-X \text { includes some } A_{a} \text {. }
$$

And secondly, for each $g: N \rightarrow N$ such that $[g]_{D} \in L:$

$$
\text { There is an a such that, for all } n, c_{A_{a}}(n) \leq g(n) \text {. }
$$

The conditions (1X) guarantee that $E$ is an ultrafilter (which was tacitly assumed in the preceding discussion) and the conditions $(2 \mathrm{~g})$ guarantee that the upper part of the associated cut includes $L$.

We construct an almost decreasing sequence of large sets $A_{a}$, satisfying all the conditions (1X) and $(2 g)$ by induction on $a<x_{1}$. As we are assuming $C H$, the conditions $(1 \mathrm{X})$ and $(2 \mathrm{~g})$ can all be arranged in a sequence of length $\boldsymbol{X}_{1}$. The $A_{a}$ 's will be defined so that $A_{\beta+1}$ satisfies the $\beta$ th condition. Assume that $A_{\beta}$ has been defined and is large for all $\beta<\alpha$, and that these $A_{\beta}$ 's are almost decreasing. The definition of $A_{a}$ is in four cases.

If $a=0$, let $A_{0}=\{(x, y) \in N \times N \mid y<g(x)\}$ for some $g$ such that $[g] \epsilon L$. If $a=\beta+1$ and the $\beta$ th condition is (1X), let $A_{a}$ be $A_{\beta} \cap X$ or $A_{\beta}-X$, whichever is large (either one will do if both are large). Note that $A_{\beta} \cap X$ and $A_{\beta}-X$ cannot both be small for then the closure of $S$ under addition would imply that their union $A_{\beta}$ is small.

If $\alpha=\beta+1$ and the $\beta$ th condition is $(2 g)$, then first define $C_{n}$ to consist of the first $g(n)$ elements of the fiber $A_{\beta} \cap p^{-1}\{n\}$ (or the whole fiber if it has fewer than $g(n)$ elements). Then set $A_{a}=\bigcup_{n} C_{n}$. It is large and satisfies (2g) because $c_{A_{a}}(n)=\min \left\{g(n), c_{A_{\beta}}(n)\right\}$.

There remains the case that $\alpha$ is a (countable) limit ordinal. We must find 
a large set $A_{a}$ that is almost included in each $A_{\beta}$. Since the sequence of $A_{\beta}$ 's is almost decreasing, the intersection of finitely many $A_{\beta}$ 's is almost equal to the last of them, and is therefore large. By rearranging the $A_{\beta}$ 's in an $\omega$-sequence and then replacing each of them by its intersection with all the preceding ones (in the new ordering), we obtain a decreasing $\omega$-sequence of large sets $B_{0} \supseteq B_{1} \supseteq$ $B_{2} \supseteq \cdots$ such that each $A_{\beta}$ includes some $B_{n}$. It will clearly suffice to find a large set $A_{a}$ almost included in each $B_{n}$. As $B_{n}$ is large and every countable subset of $L$ has a lower bound in $L$, we can find $[g] \in L$ with $g(k) \leq c_{B_{n}}{ }^{(k)}$ for all $k$ in some set $H_{n} \in D$. Without loss of generality (redefining $g$ to be 0 on $\left.N-H_{0}\right)$, we can assume $H_{0}=N$. For each $k$, let $b(k)$ be the largest $n \leq k$ such that $k \in H_{n}$. Then $b^{-1}\{0,1, \ldots, n\}$ is disjoint from the set $H_{n+1} \cap\{k \in N \mid k>n\}$ which is in $D$, so $b$ is not constant on any set of $D$. As $D$ is a $P$-point, $b$ must be finite-to-one on some $K \in D$. Let $C_{k}$ consist of the first $g(k)$ elements of $B_{b(k)} \cap p^{-1}\{k\}$ (which has at least $g(k)$ elements, by definition of $b$ and $H_{n}$ ). Then $A_{a}=\bigcup_{k} \in K C_{k}$ has cardinality function equal to $g$ on $K$, hence mod $D$, so $A_{a}$ is large. Since $C_{k} \subseteq B_{b(k)}$ and the $B_{n}$ are a decreasing sequence we have

$$
A_{a}-B_{n} \subseteq \bigcup_{b(k)<n ; k \in K} C_{k}
$$

as $b$ is finite-to-one, this is a finite union of finite sets, so $A_{a}$ is almost included in $B_{n}$. This completes the inductive construction of the $A_{a}$ 's and thus the proof of the theorem.

Proof of Theorem 3. Suppose the proper cut $(S, L)$ is associated to an irreducible map $p: E \rightarrow D$. Let $[f]_{D}$ and $[g]_{D}$ be germs whose product $[f g]$ is in $L$; we show that $[f]$ or $[g]$ is in $L$. There is an $A \in E$ such that $c_{A}(n) \leq f(n) g(n)$ for all $n$ in some set $B \in D$; replacing $A$ by $A \cap p^{-1}(B)$, we can assume $c_{A}(n) \leq f(n) g(n)$ for all $n$. Thus, each fiber $A \cap p^{-1}\{n\}$ can be partitioned into $f(n)$ or fewer sets, each of cardinality at most $g(n)$. If we let $b$ be a function which is constant on each set of such a partition but takes different values on different sets, then any subset of $A$ on which $b$ is fiberwise one-to-one must have cardinality function $\leq f$, while any subset of $A$ on which $b$ is fiberwise constant has cardinality function $\leq g$. As $p$ is irreducible, some such subset is in $E$, so $[f]$ or $[g]$ is in $L$.

Now let $\left\{\left[f_{n}\right] \mid n \in N\right\}$ be a countable subset of $L$. To find a lower bound in $L$ for this set, it will clearly suffice to find a lower bound in $L$ for any set $\left\{\left[f_{n}^{0}\right] \mid n \in N\right\}$ with $\left[f_{n}^{\prime}\right] \leq\left[f_{n}\right]$ for all $n$. Thus, we may assume, without loss of generality, that $f_{n}$ is the cardinality function of a set $A_{n} \in E$ and that $A_{n} \supseteq A_{n+1}$ for all $n$. If $\bigcap_{n \in N} A_{n} \in E$, its cardinality function gives the required lower bound, so we may assume that this intersection is not in $E$. Replacing $A_{n}$ by $A_{n}-\bigcap_{k \in N} A_{k}$, we may even suppose that the intersection is empty. Notice that the function $g_{n}$ defined by $g_{n}(x)^{2} \leq f_{n}(x)<\left(g_{n}(x)+1\right)^{2}$ satisfies $\left[g_{n}\right] \in L$ because $S$ is closed under multiplication. If, for some $n,\left[g_{n}\right]$ is a lower bound 
for all the $\left[f_{k}\right]$ 's, we are done, so suppose this is not the case. We may then replace the decreasing sequence of $f_{n}$ 's by a subsequence and assume, without loss of generality, that $\left[f_{n+1}\right]<\left[g_{n}\right]$, so $\left[f_{n+1}^{2}\right]<\left[f_{n}\right]$. Now define $b: N \rightarrow N$ by letting $b(x)$ be the least $n$ such that $x \notin A_{n}$; such an $n$ exists because $\bigcap_{n \in N} A_{n}=\varnothing$.

Case 1. $b$ is fiberwise constant on some $B \in E$. Thus, there is a function $q$ such that $b(x)=q(p(x))$ for all $x \in B$. For $x \in B \cap A_{0}=B^{\prime}$, the definition of $b$ yields $x \in A_{q p(x)-1}$. Hence, for $y \in p\left(B^{\prime}\right)$, the cardinality function of $B^{\prime}$ satisfies $c_{B}(y) \leq f_{q(y)-1}(y)$. Consider any fixed $k \in N$. If $q$ were bounded by $k$ on some set $C \in D$, then $A_{k} \cap B \cap p^{-1}(C)$ would be empty and in $E$, a contradiction. So $q(y)>k$ for all $y$ in some set $C \in D$. Then, for such $y$,

$$
c_{B}(y) \leq f_{q(y)-1}(y) \leq f_{k}(y)
$$

(as the $f_{n}$ 's form a decreasing sequence). We have thus shown that $\left[c_{B}\right]$, which is clearly in $L$, is a lower bound for the $\left[f_{k}\right]$ 's.

Case 2. $b$ is fiberwise one-to-one on some $B \in E$. Replacing $B$ by $B \cap A_{0}$, we may assume that $b$ is never zero on $B$. Consider any fixed $k \in N$. As $b$ takes only $k+1$ values (namely $1,2, \cdots, k+1$ ) on $A_{0}-A_{k+1}$ and is fiberwise oneto-one on $B \subseteq A_{0}$, we see that $\left(B-A_{k+1}\right) \cap p^{-1}\{y\}$ has at most $k+1$ elements, so $B \cap A_{k+1} \cap p^{-1}\{y\}$ must have at least $c_{B}(y)-k-1$ elements. As $f_{k+1}$ is the cardinality function of $A_{k+1}$, we have $c_{B}(y) \leq f_{k+1}(y)+k+1$, so

$$
\left[c_{B}\right] \leq\left[f_{k+1}\right]+k+1<\left[f_{k+1}\right]^{2}<\left[f_{k}\right]
$$

(because $\left[f_{k+1}\right]$ is infinite, and because we arranged that $\left[f_{k+1}^{2}\right]<\left[f_{k}\right]$ before defining $b)$. Therefore, $\left[c_{B}\right]$ is the required lower bound.

As $p$ is irreducible, one of the two cases occurs, so the $\left[f_{n}\right]$ 's are bounded from below in $L$.

To prove the converse part of the theorem, let $D$ and a cut $(S, L)$ satisfying the conditions of the thẹorem be given. As in the corresponding part of the proof of Theorem 2, we let $p=\pi_{1}$, and we obtain $E$ as the filter generated by a sequence $\left\{A_{a} \mid a<X_{1}\right\}$ of large sets (i.e. $\left[c_{A_{a}}\right] \in L$ ). This sequence will not (in general) be almost decreasing, but it will be decreasing modulo $D$, i.e. for $a<\beta, p\left(A_{\beta}-A_{\alpha}\right) \notin D$. The same conditions (1X) and $(2 \mathrm{~g})$ as in the previous proof suffice to guarantee that $E$ is an ultrafilter, $p: E \rightarrow D$, and $(S, L)$ is the associated cut. To guarantee that $p$ is irreducible, we add, for each map $f: N \times N \rightarrow N$, the condition

$$
f \text { is fiberwise one-to-one or fiberwise constant on some } A_{\alpha} \text {. }
$$

We well-order the conditions in a sequence of length $\kappa_{1}$ (using $\mathrm{CH}$ ) and define 
the $A_{a}$ 's by induction. The cases that $\alpha=0$ or $a=\beta+1$ and the $\beta$ th condition is $(1 \mathrm{X})$ or $(2 \mathrm{~g})$ are handled exactly as in the proof of Theorem 2 .

If $\alpha=\beta+1$ and the $\beta$ th condition is (3f), we let $g(n)$ be the number of values taken by $f$ on the fiber $A_{\beta} \cap p^{-1}\{n\}$, and we let $b(n)$ be the largest number of times any single value is taken by $f$ on that fiber. Thus, $A_{\beta}$ has a subset $B$, on which $f$ is fiberwise one to-one with $c_{B}=g$, and it has another subset $C$ on which $f$ is fiberwise constant with $c_{C}=b$. As $c_{A}(n) \leq g(n) b(n)$ and $S$ is closed under multiplication, either $B$ or $C$ must be large and can therefore serve as $A_{a}$.

In the case that $\alpha$ is a limit ordinal, we can repeat the argument for Theorem 2, replacing "almost" by "modulo $D$," up to and including the definition of $b$ and the observation that $b$ is not bounded on any set of $D$. We also define $C_{k}$ as before, and let $A_{a}=\bigcup_{k \in N} C_{k}$. We find that

$$
A_{a}-B_{n} \subseteq \bigcup_{b(k)<n} C_{k} \subseteq p^{-1}\{k \mid b(k)<n\},
$$

so $A_{a}$ is included in $B_{n}$ modulo $D . A_{a}$ has cardinality function $g$, so it is large. This completes the inductive construction and the proof of the theorem.

Before proving Theorem 4, we develop a few properties of weakly Ramsey ultrafilters that will be useful in that proof. Let $E$ be a weakly Ramsey ultrafilter that is not Ramsey. Thus, there is a function $p$ which is neither one-to-one nor constant on any set of $E$. We let $D=p(E)$ and observe that $D$ is nonprincipal and $p: E \rightarrow D$ is not an isomorphism. I claim that $D$ is Ramsey. To see this, let $F:[N]^{2} \rightarrow\{0,1\}=2$ be any partition of the unordered pairs of elements of $N$, and let $G:[N]^{2} \rightarrow 3$ be the partition defined by

$$
G a, b\}= \begin{cases}F\{p(a), p(b)\}, & \text { if } p(a) \neq p(b) \\ 2, & \text { otherwise. }\end{cases}
$$

Then there is an $A \in E$ such that $G$ takes only two values on $[A]^{2}$. The omitted value cannot be 2 , for $p$ is not one-to-one on any set of $E$. So $G$ takes only one of the values $\{0,1\}$ on $[A]^{2}$. This is then the only value taken by $F$ on $[p(A)]^{2}$; as $p(A) \in D$, we conclude that $D$ is Ramsey.

Next, consider any map $f$, and define a partition $G:[N]^{2} \rightarrow 3$ by

$$
G a, b\}=\left\{\begin{array}{lll}
0, & \text { if } p(a)=p(b) & \text { and } f(a)=f(b) ; \\
1, & \text { if } p(a)=p(b) \text { and } f(a) \neq f(b) ; \\
2, & \text { if } p(a) \neq p(b) .
\end{array}\right.
$$

Let $A \in E$ be such that $G$ takes only two values on $[A]^{2}$. The omitted value cannot be 2 , for $p$ is not constant on any set of $E$. If the omitted value is 0 , then $f$ is fiberwise one-to-one on $A$, while if it is 1 , then $f$ is fiberwise constant 
on $A$. We have thus shown that $p$ is irreducible. We can, however, obtain more information in the case that $f$ is fiberwise one-to-one. Define another partition $G:[N]^{2} \rightarrow 3$ by

$$
\{a, b\}=\left\{\begin{array}{lll}
0, & \text { if } p(a)<p(b) & \text { and } f(a)<f(b) ; \\
1, & \text { if } p(a)<p(b) & \text { and } f(a) \geq f(b) ; \\
2, & \text { if } p(a)=p(b) . &
\end{array}\right.
$$

As before we obtain an $A \in E$ on which $G$ omits a value, and we see that the omitted value cannot be 2 . If the omitted value were 0 and if we let $a$ be an element of $A$ for which $p(a)$ is as small as possible, then $A$ would be a subset of $p^{-1}(p\{a\}) \cup\{b \mid f(b) \leq f(a)\}$. The first term of this union is not in $E$ (for $D$ is nonprincipal), so the second term would be in $E$. As $f$ is fiberwise one-to-one on a set of $E$, we would have a set $B \in E$ on each of whose fibers $f$ is one-to-one and takes at most $f(a)+1$ values. This contradicts the fact that every set of $E$ must have an unbounded cardinality function because $p$ is not one-to-one on any set of $E$. Therefore, the omitted value must be 1 , but then, $f$ never takes the same value on two different fibers of $A$. Combining this with the fact that $f$ is fiberwise one-to-one on $A$, we conclude that $f$ is one-to-one on $A$. Applying this discussion to the case where $f$ is the identity function, we see furthermore that $p$ is a nondecreasing function on $A$; since it takes infinitely many values on $A$ (for $D$ is nonprincipal) $p$ must be finite-to-one on $A$.

Theorem 5. Let $E$ be weakly Ramsey but not Ramsey, and let $p: E \rightarrow D$ be a nonisomorpbic map to a nonprincipal ultrafilter. Then

(1) $D$ is Ramsey.

(2) $E$ is a P-point.

(3) Any function $f$ is either one-to-one, or constant, or of the form $g \circ p$ wbere $g: D \cong D^{\prime}$, on some set $E$.

Proof. The preceding discussion established (1) and that any $f$ is either oneto-one or of the form $g \circ p$ (i.e. fiberwise constant) on some set of $E$. To prove (3), we need only observe that $g$ is either an isomorphism or constant mod $D$, because $D$ is Ramsey. Since $p$ is finite-to-one, (2) follows immediately from (3).

Notice that (3) has the following model-theoretic interpretation: The only proper submodels of $E-\operatorname{prod} N$ are $p^{*}(D-\operatorname{prod} N)$ and the standard model $N$. It also shows that our initial choice of $p$ (as any nonconstant nonisomorphic map) was much less arbitrary than it seemed. Up to composition with an isomorphism $D \cong D^{\prime}$, there is only one such map.

Proof of Theorem 4. Suppose the proper cut $(S, L)$ is associated to $p: E \rightarrow D$ and $E$ is weakly Ramsey. As $(S, L)$ is proper, $p$ is neither constant nor 
one-to-one on any set of $E$, so the preceding discussion and Theorem 5 are applicable. In particular, $D$ is Ramsey and $p$ is irreducible, so, by Theorem $3, S$ is closed under addition and multiplication, and every countable subset of $L$ has a lower bound in $L$. Suppose that $2^{[f]} \in L$; we shall show that $[f] \in L$. Let $A \in E$ be such that $c_{A}(n) \leq 2^{f(n)}$ for all $n \in B \in D$; replacing $A$ by $A \cap p^{-1}(B)$, we assume the inequality holds for all $n$. By a theorem of Erdös [ 6], we can partition the set of unordered pairs from each fiber of $A$ into two parts so that there is no homogeneous set of cardinality $2 f(n)$ in the $n$th fiber; let $F_{n}:\left[A \cap p^{-1}\{n\}\right]^{2}$ $\rightarrow 2$ be such partitions. Define $G:[A]^{2} \rightarrow 3$ by

$$
G a, b\}= \begin{cases}F_{n}\{a, b\} & \text { if } p(a)=p(b)=n ; \\ 2 & \text { if } p(a) \neq p(b),\end{cases}
$$

and let $A^{\prime} \in E, A^{\prime} \subseteq A$ be such that $G$ omits a value on $\left[A^{\prime}\right]^{2}$. As $p$ is not constant on any set of $A^{\prime}$, the omitted value is not 2. Therefore, $A^{\prime} \cap p^{-1}\{n\}$ is homogeneous for the partition $F_{n}$ and must have fewer than $2 f(n)$ elements. So $2[f] \in L$, and, as $S$ is closed under addition, $[f] \in L$. Thus, $S$ is closed under exponentiation when the base is 2 ; to handle the general case, simply observe that (since $S$ is closed under multiplication)

$$
x, y \in S \Rightarrow x y \in S \Rightarrow x^{y} \leq\left(2^{x}\right)^{y}=2^{x y} \in S \text {. }
$$

To prove the converse part of the theorem, let $D$ and $(S, L)$ satisfying all the conditions be given. The construction of the required $E$ with $p=\pi_{1}$ proceeds exactly as in the proof of Theorem 2, except that we insure that $E$ is weakly Ramsey by adding to $(1 \mathrm{X})$ and $(2 \mathrm{~g})$ the following condition for each partition $F:[N]^{2} \rightarrow 3:$

There is an a such that $F$ takes only two values on $\left[A_{a}\right]^{2}$.

To complete the proof, we need only show how to satisfy these extra conditions in the inductive construction of the $A_{\alpha}$ 's. Suppose a large set $A_{\beta}$ is given and the $\beta$ th condition is (3F); we construct a large subset $A_{\beta+1}$ of $A_{\beta}$ such that $F$ takes only two values on $\left[A_{\beta+1}\right]^{2} . A_{\beta+1}$ will be obtained by cutting down $A_{\beta}$ in a sequence of steps.

Step 1. In each fiber $A_{\beta} \cap p^{-1}\{n\}$, choose a subset $B_{n}$ such that $F$ takes either only the values $\{0,1\}$ or only the value 2 on $\left[B_{n}\right]^{2}$. By well-known estimates in connection with Ramsey's theorem, $B_{n}$ can be taken to have cardinality at least $1 / 2 \log _{2} c(n)$ where $c$ is the cardinality function of $A_{\beta}$ (see [7]). If $F$ takes the values 0 and 1 on $\left[B_{n}\right]^{2}$, extract yet smaller subsets on which $F$ is homogeneous; otherwise, keep the $B_{n}$ 's you have. These sets will have cardinality at least $1 / 2\left(\log _{2} \log _{2} c(n)-1\right){ }^{n}$ As $S$ is closed under exponentiation, the union $A^{\prime}$ 
of these sets is large, and, for $a, b \in A^{\prime}$ and $p(a)=p(b), F\{a, b\}$ depends only on $p(a)$, say $F\{a, b\}=f(p(a))$.

Step 2. As $D$ is an ultrafilter and $f$ takes only three values, find a set $B \in D$ on which $f$ is constant, and let $A^{\prime \prime}=A^{\prime} \cap p^{-1}(B)$. Being equal to $A^{\prime} \bmod D, A^{\prime \prime}$ is large, and there is a single number $\xi$ such that $F\{a, b\}=\xi$ whenever $a$ and $b$ are in the same fiber of $A^{\prime \prime}$.

Step 3. Let $c^{\prime \prime}$ be the cardinality function of $A^{\prime \prime}$, and let $e(n)=\sum_{k=0}^{n} c^{\prime \prime}(k)$. For $a<b \in N$, let

$$
G a, b\}= \begin{cases}0, & \text { if } c^{\prime \prime}(b) \leq 9^{e(a)} \\ 1, & \text { if } c^{\prime \prime}(b)>9^{e(a)} .\end{cases}
$$

and, as $D$ is Ramsey, let $B \in D$ be homogeneous for this partition. Let $a$ be the first element of $B$. As $c^{\prime \prime}$ is not bounded by any finite number on $B$ (for $A^{\prime \prime}$ is large), in particular not by $9^{e(a)}$, the value of $G$ on $[B]^{2}$ must be 1 . Let $A^{\prime \prime \prime}=A^{\prime \prime} \cap p^{-1}(B)$. If we let $c^{\prime \prime \prime}$ be its'cardinality function and $e^{\prime}(n)=\sum_{k=0}^{n} c^{\prime \prime \prime}(k)$, then $a<b \in B$ implies $c^{\prime \prime \prime}(b)>g^{e^{\prime}(a)}$.

Step 4. For each $x \in A^{\prime \prime \prime}$, let $\psi(x)$ be the partial function, with domain $\left\{y \in A^{\prime \prime \prime} \mid p(y)<p(x)\right\}$, defined by $\psi(x)(y)=F\{y, x\}$. If $b \in B$ (where $B$ is as in Step 3), $a$ is the last element of $B$ before $b$, and $x \in A^{\prime \prime \prime} \cap p^{-1}\{b\}$, the domain of $\psi(x)$ has $e^{\prime}(a)$ elements $(0$, if $b$ is the first element of $B)$, so there are $3^{e^{\prime}(a)}$ possibilities for $\psi(x)$. As this number is less than $\sqrt{c^{\prime \prime \prime}(b)}$, there are at least $\sqrt{c^{\prime \prime \prime}(b)}$ elements $x \in A^{\prime \prime \prime} \cap p^{-1}\{b\}$ all yielding the same function $\psi(x)$. As $S$ is closed under multiplication and $A^{\prime \prime \prime}$ is large, we can extract a large subset $A^{(4)}$ such that $\psi(x)$ is the same for all $x$ in any fiber of $A^{(4)}$, and we can write $\psi(x)=$ $\phi(p(x))$ for all $x \in A^{(4)}$. By definition of $\psi$, we have $F\{x, y\}=\phi(p(y))(x)$ whenever $x, y \in A^{(4)}$ and $p(x)<p(y)$.

Step 5. For each $x \in A^{(4)}$ and $b \in B=p\left(A^{(4)}\right)$ such that $p(x)<b$, let $\phi_{x}(b)=\phi(b)(x)$. Then $\phi_{x}$ maps a set in $D$ into 3 , so it is constant on some $C_{x} \in D$; let $\phi_{x}^{*}$ be the value of $\phi_{x}(b)$ for all $b \in C_{x}$. For $a<b \in B$, let

$$
G a, b\}= \begin{cases}0, & \text { if } b \in C_{x} \text { for all } x \in A^{(4)} \cap p^{-1}\{a\} ; \\ 1, & \text { otherwise. }\end{cases}
$$

Let $C \in D, C \subseteq B$ be homogeneous for this partition, and let $a$ be the first element of $C$. As there are only finitely many $x \in A^{(4)} \cap p^{-1}\{a\}$ and each $C_{x}$ is in $D$, we see that the value of $G$ on $[C]^{2}$ cannot be 1 , so it is 0 . Let $A^{(5)^{x}}=$ $A^{(4)} \cap p^{-1}(C)$. Thus, if $a, b \in A^{(5)}$ and $p(a)<p(b)$ then $p(b) \in C_{a}$, so

$$
F\{a, b\}=\phi_{a}(p(b))=\phi_{a}^{*} \text {. }
$$


Step 6. The function $a \mapsto \phi_{a}^{*}$ maps each fiber into 3. As $S$ is closed under addition and $A^{(5)}$ is large, we can find a large subset $A^{(6)}$ such that this function is fiberwise constant on $A^{(6)}$. Thus, for $a, b \in A^{(6)}$ and $p(a)<p(b)$, we have for a certain function $b$.

$$
F\{a, b\}=b(p(a))
$$

Step 7. As $b$ maps $N$ into 3 , it is constant on some set $K \in D$; let its value on $K$ be $\eta$. We set $A_{\beta+1}=A^{(6)} \cap p^{-1}(K)$, and observe that, if $a, b \in A_{\beta+1}$ and $p(a) \neq p(b)$, then $F\{a, b\}=\eta$. On the other hand, if $a$ and $b$ are in the same fiber of $A_{\beta+1}$, then, by Step $2, F\{a, b\}=\xi$. Thus, $F$ takes only the values $\xi$ and $\eta$ on $\left[A_{\beta+1}\right]^{2}$. As $A_{\beta+1}$ is large (since all $A^{(k)}$ were large), the proof is complete.

Finally, let us consider what happens to our theorems when improper cuts $(S, L)$ are permitted. Trivial modification of the proof of Theorem 1 yields the result that $(\{0\}, D-\operatorname{prod} N-\{0\})$ and $(D-\operatorname{prod} N, \varnothing)$ are the only improper cuts associated to any maps $p: E \rightarrow D$. The former is associated to isomorphisms and the latter to maps that are not finite-to-one on any $A \in E$. Clearly, then, the latter cannot occur when $E$ is a $P$-point unless $D$ is principal. The former can occur with $E$ a $P$-point, if and only if $D$ is a $P$-point. Both types of improper cuts can be associated to irreducible maps if $\mathrm{CH}$ holds. Of course, any isomorphism is irreducible. Given $D$, we obtain an irreducible map $\pi_{1}: E \rightarrow D$ which is not finite-to-one on any set of $E$ by letting $E$ be the product $D \cdot F=\{A \subseteq N \times N \mid$ $\left.\left\{n \mid \pi_{2}\left(A \cap \pi_{1}^{-1}\{n\}\right) \in F\right\} \in D\right\}$ for any Ramsey ultrafilter $F$. The irreducibility of $\pi_{1}$ follows from part (2) of the definition of Ramsey ultrafilters. The improper cut ( $\{0\}, D$-prod $\{0\})$ is associated to a map $p: E \rightarrow D$ with $E$ weakly Ramsey iff $D$ is weakly Ramsey; the other improper cut $(D-\operatorname{prod} N, \varnothing)$ is associated to such a $p$ iff $D$ is principal.

\section{REFERENCES}

1. A. Blass, Orderings of ultrafilters, Thesis, Harvard University, Cambridge, Mass., 1970.

2. - The Rudin-Keisler ordering of P-points, Trans. Amer. Math. Soc. 179 (1973), 145-166.

3. D. Booth, Ultrafilters on a countable set, Ann. Math. Logic $2(1970 / 71)$, no. 1, 1-24. MR 43 \#3104.

4. G. Choquet, Construction d'ultrafiltres sur N, Bull. Sci. Math. (2) 92 (1968), 4148. MR 38 \#2722.

5. Deux classes remarquables d'ultrafiltres sur N, Bull. Sci. Math. (2) 92 (1968), 143-153. MR 38 \#5154.

6. P. Erdös, Some remarks on the theory of graphs, Bull. Amer. Math. Soc. 53 (1947), 292-294. MR 8, 479.

7. P. Erdös and G. Szekeres, A combinatorial problem in geometry, Compositio Math. 2 (1935), 463-470.

8. T. Frayne, A. Morel and D. Scott, Reduced direct products, Fund. Math. 51 (1962/ 63), 195-228. MR $26 \# 28$. 
9. H. J. Keisler, Ultraproducts and saturated models, Nederl. Akad. Wetensch. Proc. Ser. A 67 = Indag. Math. 26 (1964), 178-186. MR 29 \#5745.

10. M. E. Rudin, Partial orders on the types in $\beta N$, Trans. Amer. Math. Soc. 155 (1971), 353-362. MR 42 \$859.

11. W. Rudin, Homogeneity problems in the theory of Čech compactifications, Duke Math. J. 23 (1956), 409-419. MR 18, 324. 48104

DEPARTMENT OF MATHEMATICS, UNIVERSITY OF MICHIGAN, ANN ARBOR, MICHIGAN 